\title{
Author Correction: A compact multi-planet system around a bright nearby star from the Dispersed Matter Planet Project
}

D. Staab, C. A. Haswell (D), J. R. Barnes (D), G. Anglada-Escudé, L. Fossati (D, J. P. J. Doherty, J. Cooper, J. S. Jenkins, M. R. Díaz and M. G. Soto

Correction to: Nature Astronomy https://doi.org/10.1038/s41550-019-0974-x, published online 23 December 2019.

In the version of this Article originally published, Supplementary Fig. 4 was missing three 55Cnc data points in each panel. The Supplementary Information has now been replaced to correct this.

Published online: 13 March 2020

https://doi.org/10.1038/s41550-020-1074-7

(๑) The Author(s), under exclusive licence to Springer Nature Limited 2020 